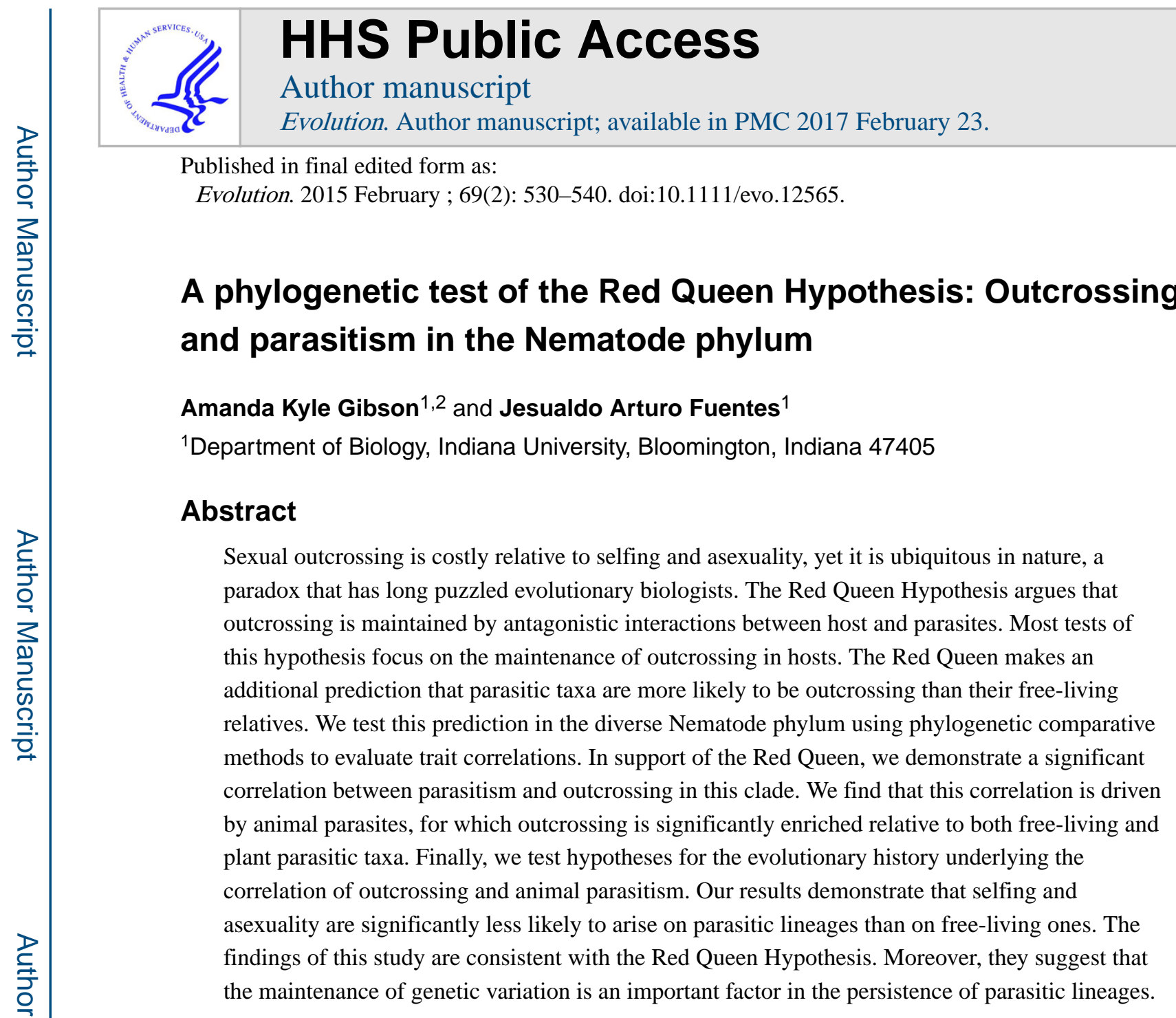

\title{
Keywords
}

Asexual reproduction; continuous-time Markov model; host-parasite coevolution; selfing; sexual outcrossing; stochastic character mapping

Outcrossing, the fusion of gametes from two different individuals, is the most prominent reproductive strategy in eukaryotes. Uniparental modes of inheritance, including selffertilization and parthenogenesis, are in contrast quite rare (Bell 1982; Suomalainen et al. 1987; Dacks and Roger 1999; Billiard et al. 2012). Yet, outcrossing carries significant costs. Theory predicts that these costs accrue as either a significantly depressed per-capita growth

2 amakgibs@indiana.edu.

Supporting Information

Additional Supporting Information may be found in the online version of this article at the publisher's website:

Figure S1. Estimates of correlation in subsampled relative to true phylogenies under different sampling schemes.

Table S1. Species-level detail for character assignments.

Table S2. Number of character changes estimated under parsimony and stochastic character mapping.

Table S3. Summary of results of stochastic character mapping.

Table S4. Summary of results of the discrete model for correlated evolution of outcrossing and animal parasitism.

Table S5. Mean and variance of correlation estimates from true and subsampled phylogenies under random and biased sampling.

Table S6. Likelihood ratios and the significance of dependent evolution in true and subsampled phylogenies. 
rate of outcrossing lineages relative to uniparental lineages or as a reduction in relatedness between parent and offspring (Williams 1975; Maynard Smith 1978; Charlesworth 1980; Lively and Lloyd 1990). A paradox thus emerges: how can the prominence of outcrossing be reconciled with its costs?

The Red Queen Hypothesis offers a potential solution. It proposes that antagonistic coevolution between interacting species selects for the maintenance of outcrossing. If coevolving parasites adapt to specifically infect the most common genotypes in a host population, then rare host genotypes gain a fitness advantage by evading parasitism (Haldane 1949; Jaenike 1978). Outcrossing allows for the production of offspring with rare genotypes, whereas prolonged periods of uniparental reproduction propagate genetically uniform lineages (Hamilton 1980; Hamilton et al. 1990). The Red Queen thus predicts that outcrossing should be maintained in the presence of virulent coevolving parasites. Many empirical studies have supported this prediction by demonstrating that host-parasite coevolution explains the observed distribution of outcrossing in hosts: outcrossing is common in environments or host taxa in which parasite pressure is high (e.g., the tropics, long-lived species; Bell 1982; Burt and Bell 1987; Lively 1987; Verhoeven and Biere 2013; Wilson and Sherman 2013).

Similarly, the Red Queen predicts that outcrossing should be maintained in the coevolving parasites themselves (Bell 1982). Just as hosts are under selection to evade parasitism through the production of rare genotypes, parasites are under equivalent or greater selection to infect their ever-changing host population (Howard and Lively 2002; Galvani et al. 2003; Salathé et al. 2008; King et al. 2011). Parasites continually degrade their environment (the host population) by decreasing the frequency of the common host lineages to which they are adapted. Thus, a common parasite genotype with high fitness is predicted to deplete its host lineage and suffer low fitness in later generations. In contrast, a rare parasite genotype has a greater probability of infecting alternate host genotypes and thus gains a fitness advantage. Few empirical and theoretical studies have investigated this prediction (although see Bell 1982; Howard and Lively 2002; Zhan et al. 2007). A related prediction argues that outcrossing is favored in vertebrate parasites because of selection pressure exerted by the rapidly coevolving adaptive immune system. This prediction has received little support thus far (Gemmil et al. 1997; Lythgoe 2000; West et al. 2001; although see Galvani et al. 2001, 2003).

Bell first formulated the parasite-centric prediction of the Red Queen in his 1982 book The Masterpiece of Nature. He argued that under this hypothesis, outcrossing should be more common in parasitic taxa than in their free-living relatives. This prediction was indirectly supported by the difficulty of finding taxa with which to address it: many eukaryotic parasitic groups are invariably outcrossing (e.g., phylum Acanthocephala, subclass Pentastomida; Bell 1982), impeding a comparative approach. Bell (1982) proposed the Nematode phylum as a uniquely diverse taxon for comparative studies. By comparing the reproductive mode and ecology of different nematode families, Bell (1982) offered tentative support for the Red Queen: he found that outcrossing is common in families that parasitize animals, but less so in plant parasitic and free-living families. 
A rigorous evaluation of Bell's (1982) prediction that outcrossing is more common in parasitic taxa than in their free-living relatives requires a phylogenetic comparison that accounts for the role of shared ancestry in explaining trait distributions. The tools necessary for this phylogenetic approach were not available at the time of publication of The Masterpiece of Nature. Since then, molecular and phylogenetic resources have become available for the Nematoda. Using these tools, studies have identified multiple transitions from free living to parasitism (Blaxter et al. 1998; Dorris et al. 1999; De Ley 2006; van Megen et al. 2009) and from outcrossing to uniparental reproduction in the phylum (Kiontke et al. 2004, 2011; Kiontke and Fitch 2005; Cutter et al. 2008; Denver et al. 2011).

Here, we take advantage of these resources to further test the Red Queen's prediction that outcrossing should be more common in parasitic species than in their free-living relatives. We use a recent phylogeny of the Nematode phylum (Meldal et al. 2007) to make a finescale species-level comparison of parasitic and free-living taxa while accounting for shared ancestry. Adding to Bell's preliminary results, we find that the Red Queen Hypothesis successfully explains the macroevolutionary distribution of outcrossing. Although outcrossing is maintained in lineages of parasitic nematodes, notably in animal parasitic lineages, free-living lineages are susceptible to invasion by uniparental modes of reproduction.

\section{Methods PHYLOGENY}

Meldal provided the phylogenetic reconstructions from Meldal et al. (2007), which were based upon small subunit ribosomal DNA for 212 taxa distributed across the phylum. Bayesian inference in MrBayes version 3.1.2 (Huelsenbeck and Ronquist 2001) produced 2700 trees.

For comparative analyses, we removed the closely related marine clades Desmorida, Chromadorida, and Monhysterida ( $n=48$ taxa). Marine taxa are poorly studied (Meldal et al. 2007), and reproductive mode was difficult to ascertain. An additional two taxa, Calyptronema maxweberi and one identified only as a marine Tylenchid, were removed due to lack of character data. Marine nematodes should be the focus of future study: they are thought to be largely outcrossing (Bell 1982).

Pruning in Mesquite version 2.75 (Maddison and Maddison 2011) produced a phylogeny of 162 species with Turbanella cornuta as an outgroup. Pruned trees were made ultrametric using maximum-likelihood optimization with the package phangorn version 1.99-5 (Schliep 2011) for $R$ version 3.0.2 (R Core Team 2013). For this purpose, we used the original sequence alignments and the TIM2+I+G model, which was selected in jModelTest under the Bayesian information criterion with the BIONJ setting (Guindon and Gascuel 2003; Darriba et al. 2010).

\section{CHARACTER ASSIGNMENTS}

For taxa in their phylogeny, Meldal et al. (2007) reported lifestyle, which is the term used here to distinguish free-living from parasitic taxa. We adopted their lifestyle 
characterizations, with two exceptions supported by the literature. Reproductive mode was determined through literature reviews, personal communication with experts, and searches of databases (Plant and Insect Parasitic Nematodes [http://nematode.unl.edu/]; Nemaplex [Ferris 1999]; Worm Bazaar [Carter and De Ley 2005]; WormBook [http:// www.wormbook.org/chapters/www_quicktourdiversity/quicktourdiversity.html]; Worm Systematic Resource Network [Fitch 1998]). For taxa identified by genus alone, character states were assigned based upon the genus's type or best characterized species.

For reproductive mode, taxa were classified as having an outcrossing or uniparental mode. Uniparental encompasses both parthenogenesis (strict asexuality) and androdioecy (hermaphrodites self-fertilize and occasionally outcross with rare males). For many taxa, male frequency was the primary factor in determining reproductive mode, with rare or absent males indicating uniparental reproduction (Maupas 1900; Triantaphyllou and Hirschmann 1964). Because male rarity cannot distinguish parthenogenesis from androdioecy, these two modes were merged under uniparental reproduction (see Table S1 for details). Theory suggests that the benefits of outcrossing may be obtained by even the rare outcrossing observed for androdioecious nematodes (Hurst and Peck 1996; Agrawal and Lively 2001; Barrière and Félix 2005). Thus, the combination of androdioecy and parthenogenesis is conservative for evaluating our hypothesis.

Ancestral states were estimated with stochastic character mapping (Nielsen 2002; Huelsenbeck et al. 2003) in SIMMAP version 1.5 (Bollback 2006) using the posterior distribution of trees.

\section{CORRELATED EVOLUTION}

We used two different phylogenetic comparative methods to test the hypothesis that outcrossing and parasitism are correlated. Each method allows for different analyses, and support for a pattern is greatly strengthened when similar results are obtained using approaches with distinct theoretical frameworks. Firstly, we used stochastic character mapping to measure the correlation between outcrossing and parasitism, a general classification encompassing both plant and animal parasitism. Because this approach allows multistate characters, we also tested the more specific hypothesis that outcrossing and animal parasitism are correlated. Secondly, we used the Discrete method to determine if transitions in lifestyle and reproductive mode are correlated (Pagel 1994). We restricted this analysis to free-living and animal parasitic taxa, excluding plant parasites. This approach estimates transition rates between character states, so we also compared transition rates to test two hypotheses for the observed evolutionary patterns. Basic deviations in character distributions were investigated using chi-square tests in $\mathrm{R}$.

Stochastic character mapping-Stochastic character mapping was implemented in SIMMAP. This method creates stochastic character maps by sampling the posterior distribution of trees and model parameters. Stochastic character maps provide a posterior distribution of character histories with which to quantify character correlation (Nielsen 2002; Huelsenbeck et al. 2003; Bollback 2006). The method measures the observed frequency of co-occurrence of states $i$ and $j$ across character maps and their expected co- 
occurrence given the frequency of each state. The statistic $d$ is the deviation of observed from expected. Positive values indicate greater co-occurrence than expected, whereas negative values indicate less than expected. Significance is determined by sampling $d$ from character maps constructed under the assumption that character states are not associated. The probability of the observed $d$ value is measured against this null distribution (Huelsenbeck et al. 2003; Bollback 2006).

SIMMAP accounts for uncertainty in modeling character evolution by assigning priors on the parameters for each trait (Schultz and Churchill 1999; Bollback 2006). Prior parameters were obtained using SIMMAP's Markov chain Monte Carlo (MCMC) analysis to generate a posterior distribution for the overall rate of character evolution (under a gamma prior) and the bias parameter (under a beta prior for two-state characters or an empirical prior for threestate characters). Posterior distributions were analyzed in R using the SIMMAP script (http://www.simmap.com/pgs/priors.html) to obtain the best-fitting parameters. Prior parameters were determined independently for each analysis using the consensus phylogeny.

Analyses of character correlation were performed using the 2700 trees, rooted and with outgroup excluded. A total of 100 samples, prior draws, and predictive samples for significance measures were taken. Specific analyses are outlined in Table S3.

Discrete method-The Discrete method (Pagel 1994) was implemented in BayesDiscrete within BayesTraits version 1.0 (Pagel and Meade 2006). BayesDiscrete tests if the evolution of two binary traits is best explained by a model of dependent or independent evolution. Under dependent evolution, transitions in lifestyle depend upon the state of reproductive mode, and vice versa. Under independent evolution, transitions in lifestyle are independent of reproductive mode, and vice versa (Pagel 1994; Pagel and Meade 2006). The Bayesian version allows for two comparisons of competing models (dependent vs. independent). First, we used a Bayes factor of the marginal likelihood of competing models (Kass and Raftery 1995). The marginal likelihood is approximated by the harmonic mean of the likelihoods in a very long Markov chain. Second, we compared the proportion of visits made to independent versus dependent models under the dependent mode of the reversiblejump MCMC. Of the 21,146 models possible under this analysis, $51(0.24 \%)$ are consistent with independent evolution. Therefore, if $0.24 \%$ of visits by the reversible-jump MCMC analysis are to independent models, the odds of dependent versus independent models of evolution are equivalent (Pagel and Meade 2006).

Plant parasitic lineages were excluded from the dataset so as to test correlated evolution of animal parasitism and outcrossing. The 2700 trees were rooted and outgroup excluded. Each analysis was run for 100,050,000 iterations, with a burn-in of 50,000 iterations, sampling every 300 iterations. A reversible-jump gamma hyperprior was used, with parameters seeded from uniform distributions on the interval 0-10. A rate deviation parameter of 9 was chosen to obtain an average acceptance rate of $20-40 \%$. Because the harmonic mean of the likelihood may have very large variance and can thus be unstable, five runs of both the independent and dependent analyses were performed (Newton and Raftery 1994; Pagel and Meade 2006; Raftery et al. 2006). 
If dependent models of evolution are supported, the underlying transition rate parameters of the dependent analysis can be examined (Pagel and Meade 2006). We tested specific hypotheses by comparing the posterior distributions of the following transition rates: $\mathrm{q}_{13}$ with $\mathrm{q}_{24}$, outcrossing to uniparental reproduction on free-living and animal parasitic backgrounds, respectively; $\mathrm{q}_{13}$ with $\mathrm{q}_{12}$, free living to parasitism on an outcrossing background; and $\mathrm{q}_{12}$ with $\mathrm{q}_{34}$, free living to parasitism on outcrossing and uniparental backgrounds, respectively.

Reversals from uniparental reproduction to outcrossing may be rare, even impossible (Igic et al. 2006; Goldberg and Igic 2008). An additional analysis was therefore conducted with the transition rate from uniparental reproduction to outcrossing $\left(\mathrm{q}_{31}\right.$ and $\mathrm{q}_{42}$ in dependent models; beta1 in independent) restricted to 0 . Each analysis was run for 1,000,100,000 iterations, with a burn-in of 50,010,000 iterations, sampling every 600 iterations. A gamma hyperprior was used, with parameters seeded from uniform distributions on the interval 0-5. A rate deviation parameter of 5 was chosen. Specific analyses are outlined in Table S4.

Both comparative methods described above rely upon a Markov process that Maddison and FitzJohn (2014) argue is flawed. The crux of the problem lies in the assumption of the Markov process that small branch segments are independent. They particularly cite as problematic datasets in which transitions in a character are rare and/or concentrated in a single lineage. In the supplement, we therefore report the methods and results for estimation of transition numbers. Maddison and FitzJohn (2014) also cite nonrandom sampling of characters as a contributing problem. We address this issue in the supplement by measuring correlations under simulations of different sampling schemes.

\section{Results}

\section{ANCESTRAL STATES FOR REPRODUCTIVE MODE AND LIFESTYLE}

The phylogeny used in comparative analyses was modified from Meldal et al. (2007) and comprises 162 nematode species (Fig. 1). Character states are summarized in Table 1 and detailed in Table S1. Ancestral states were estimated on the posterior distribution of trees using stochastic character mapping. For lifestyle, a free-living ancestor is strongly supported (probability: 99.5\%). For reproductive mode, outcrossing as the ancestral state is weakly supported (probability: 64.2\%). We find support for multiple transitions between states for both lifestyle and reproductive mode (Table S2).

\section{CORRELATION BETWEEN OUTCROSSING AND PARASITISM}

Stochastic character mapping-In our dataset, the proportion of parasitic species that are outcrossing significantly exceeds that predicted by the joint probabilities of parasitism and outcrossing in the dataset $\left(\chi^{2}=7.91, \mathrm{df}=1, P=0.005\right)$. This excess of outcrossing holds when accounting for phylogeny: outcrossing and parasitism are significantly positively associated in the evolutionary history of the Nematoda ( $d=0.011, P=0.02$; Table S3).

The excess of outcrossing in parasitic species is driven by animal parasites: $100 \%$ are obligate outcrossers $(n=43)$ or have an outcrossing stage in their life cycle $(n=2)$. In contrast, only $60.5 \%$ of plant parasites are outcrossing, which is equivalent to the proportion 
observed in free-living taxa $\left(60.8 \% ; \chi^{2}=0, \mathrm{df}=1, P=1\right)$. Indeed, a test for correlated evolution contrasting taxa that are free living, parasitic on animals, or parasitic on plants finds that outcrossing is significantly negatively correlated with free living $(d=-0.009, P<$ $0.001)$, significantly positively correlated with animal parasitism $(d=0.011, P<0.001)$, and not correlated with plant parasitism $(d=-0.002, P=0.19)$. The correlation of outcrossing and animal parasitism is unchanged when 16 species that facultatively associate with animal hosts are treated as animal parasites $(d=0.010, P<0.001)$. The lack of correlation between outcrossing and plant parasitism is similarly unchanged when 14 taxa that are questionably reported as plant parasites are treated as free living $(d=0.001, P=0.31)$. The results are also insensitive to assignment of Strongyloides ratti and Heterorhabditis bacteriophora as outcrossing or uniparental (Tables 1 and S3).

Discrete method-We then used the Discrete method to determine if evolutionary transitions in lifestyle and reproductive mode are correlated. Given the above results, we excluded plant parasites and limited this analysis to free-living and animal parasitic taxa. This approach further supports correlated evolution of outcrossing and animal parasitism. The estimate of the marginal likelihood of dependent models of evolution, in which evolutionary transitions in lifestyle may depend upon reproductive mode and vice versa, consistently and strongly exceeds that of models of independent evolution (average BF = 14.14; Table S4). The dependent mode of the reversible-jump MCMC analysis can visit both dependent and independent models of character evolution. Yet, this analysis visited independent models less than $0.0001 \%$ of the time, which is lower than the $0.24 \%$ of visits to independent models predicted if independent and dependent models were equally likely. This further supports dependent, correlated evolution.

\section{EVOLUTIONARY MECHANISMS UNDERLYING THE CORRELATION}

We now test two hypotheses for the excess of outcrossing in animal parasites. First, uniparental reproduction may evolve more readily in free-living relative to animal parasitic lineages. Second, animal parasitism may evolve more readily in outcrossing relative to uniparental lineages. Both of these hypotheses are consistent with ancestral state reconstructions here and in prior studies suggesting parasitism and uniparental reproduction as derived states.

Our previous analysis (contrasting free-living and animal parasitic taxa) demonstrates significant support for dependent over independent models of evolution. This allows for further investigation of the dependent models, specifically of the evolutionary transitions underlying correlated evolution. We therefore investigated the transition rate matrix of the dependent models to test our two hypotheses for the observed excess of outcrossing in animal parasites.

Hypothesis 1: Uniparental reproduction evolves more frequently in free-living relative to animal parasitic lineages-This hypothesis predicts that the transition rate from out-crossing to uniparental reproduction is larger on a free-living $\left(\mathrm{q}_{13}\right)$ than on an animal parasitic ( $\mathrm{q}_{24}$ ) background (Fig. 2D). Comparison of transition rates under the dependent model of evolution demonstrates significant support for this hypothesis: $q_{13}$ 
exceeds $\mathrm{q}_{24} 96.0 \%$ of the time, on average, by a large magnitude (average 16.17; Fig. 2A). This result holds when the model is specified to prevent reversals from uniparental reproduction to outcrossing (Table S4). Moreover, the evolution of reproductive mode, rather than of lifestyle, determines the observed evolutionary patterns: transition rates for reproductive mode ( $\mathrm{q}_{13}$ : outcrossing to uniparental) exceed those for lifestyle $\left(\mathrm{q}_{12}\right.$ : free living to animal parasitism) $96.1 \%$ of the time on average (average magnitude 15.57; Fig. 2B). This finding is consistent with Hypothesis 1 .

Hypothesis 2: Animal parasitism evolves more frequently in out-crossing relative to uniparental lineages-This hypothesis predicts that the transition rate from free living to animal parasitism is larger on an outcrossing $\left(\mathrm{q}_{12}\right)$ than on a uniparental $\left(\mathrm{q}_{34}\right)$ background. This hypothesis is not supported. Transition rates to animal parasitism on either background are low and statistically indistinguishable $\left(\mathrm{q}_{12}>\mathrm{q}_{34} 34.7 \%\right.$ of the time on average; Fig. 2C).

\section{Discussion}

In this study, we test the Red Queen Hypothesis's prediction that outcrossing should be more common in parasitic species than in their free-living relatives. We revisit Bell's (1982) investigation of the distribution of outcrossing and parasitism in the Nematode phylum using phylogenetic comparative tools. The results corroborate Bell's findings: there is a significant positive correlation between outcrossing and parasitism. Also consistent with Bell's results, we find that the relationship between outcrossing and parasitism is limited to animal parasites, with no correlation between plant parasitism and outcrossing. Accordingly, we tested hypotheses for the evolutionary mechanisms generating an excess of outcrossing in animal parasites. Our findings suggest that animal parasitic lineages are more resistant to invasion by uniparental strategies than are free-living lineages. These results strongly support the Red Queen Hypothesis as an explanation for the macroevolutionary distribution of outcrossing in the Nematoda.

Our phylogenetic findings corroborate prior studies of the Red Queen. Of most direct relevance is a theoretical study by Howard and Lively (2002) in which coevolution with hosts maintained outcrossing in parasites, although only in combination with mutation accumulation in clonal parasite lineages. Indirect support also comes from empirical studies of microbial experimental evolution. Coevolution of the bacteria Bacillus thuringiensis with nematode hosts resulted in bacterial populations with greater genetic diversity and more frequent horizontal gain of toxin genes, which are likely involved in host interaction (Schulte et al. 2010, 2013). The Red Queen Hypothesis has also been extended to rates of evolution, with the prediction that antagonistic coevolution leads to accelerated molecular evolution (Van Valen 1974; Hedrick 1994; Fischer and Schmid-Hempel 2005; Obbard et al. 2006). Paterson et al. (2010) demonstrated that, relative to phage evolution alone, coevolution of bacteriophage $\Phi 2$ with its host, resulted in significantly higher rates of molecular evolution for the phage, most notably at loci implicated in host interaction. These microevolutionary results support our macroevolutionary finding that the persistence of parasitic lineages requires forces that maintain genetic variation. 
Interestingly, this finding is driven by animal parasites, with plant parasites showing no excess of outcrossing relative to free-living taxa. The occurrence of uniparental reproduction in nema-tode plant parasites has been previously noted (Triantaphyllou and Hirschmann 1964; Bell 1982; Castagnone-Sereno 2006; Castagnone-Sereno and Danchin 2014). We here propose hypotheses to explain this pattern. First, outcrossing in parasites could be maintained not by coevolution with hosts per se, but rather by coevolution with the adaptive immune systems of vertebrate hosts. Prior studies have not supported this hypothesis (Gemmil et al. 1997; Lythgoe 2000; West et al. 2001), excepting Galvani et al.'s (2003) theoretical demonstration that sexual populations of helminths can resist invasion by asexual mutants. In their study, the advantage of sexual populations stems from their ability to evade host immunity by maintaining strain diversity, which is stochastically lost in asexual parasite populations. Our results are also consistent with this hypothesis: the association of outcrossing and parasitism is present in animal parasites, the vast majority of which parasitize vertebrates, and absent in taxa parasitizing plants. The animal parasites in our dataset that parasitize invertebrates are outcrossing but are too rare $(n=4)$ to provide a valid contrast with vertebrate parasites. Additional sampling of taxa parasitizing invertebrates would allow for a test of the coevolving vertebrate immune system as a force maintaining outcrossing

Second, polyphagous, agricultural pests are overrepresented among nematode plant parasites. Research in scale insects has demonstrated that asexual reproduction is more common in species that are polyphagous and/or pests (Ross et al. 2013). A broad host range may be linked with weak, nonspecific coevolutionary interactions between hosts and parasites that fail to maintain outcrossing (Thompson 1999; Lajeunesse and Forbes 2002). This hypothesis predicts a larger host range for uniparental relative to outcrossing parasites. Alternately, Ross et al. (2013) attribute the relationship between polyphagy, pest status, and uniparental reproduction to population size. Large effective population sizes $\left(N_{e}\right)$ of pest and/or polyphagous species may facilitate the persistence of uniparental lineages by reducing their probability of extinction by various forces (e.g., mutation accumulation, HillRobertson effects). Further research is required to properly contrast $N_{e}$ of uniparental and outcrossing parasitic nematodes (Nadler 1995; Criscione and Blouin 2005).

Hypotheses based upon $N_{e}$ present alternatives to the Red Queen Hypothesis (Muller 1964; Lynch et al. 1993; Otto and Barton 2001; Keightley and Otto 2006; Otto 2009; Hartfield et al. 2010, 2012), although these are not mutually exclusive (Howard and Lively 1994; Lively and Morran 2014). Indeed, prior theory on the maintenance of outcrossing in parasites argues for a combined role of host-parasite coevolution and forces that characterize finite populations (e.g., mutation accumulation [Howard and Lively 2002], stochastic extinction [Galvani et al. 2003]). Other forces may also influence the distribution of outcrossing in the Nematoda. Although the Red Queen offers an explanation for the short-term maintenance of outcrossing, limited adaptive potential and thus reduced diversification of uniparental lineages may contribute to outrossing's long-term persistence (Fisher 1930; Muller 1932; Maynard Smith 1978; Nunney 1989; Goldberg et al. 2010; de Vienne et al. 2013). Selection for reproductive assurance has also been hypothesized to explain uni-parental reproduction in taxa that inhabit unstable environments or disperse widely (e.g., androdioecy in freeliving rhabditid nematodes; Baker 1955; Pannell 2002; Weeks et al. 2006). 
Our results are consistent with the prediction of the Red Queen, and thus we cannot falsify this major hypothesis for the maintenance of outcrossing. We do acknowledge three future improvements that would test the robustness of our results. First, current tests of correlated evolution cannot account for speciation and extinction rates, which may differ between reproductive modes (Fisher 1930; Muller 1932; Maynard Smith 1978; Nunney 1989; Goldberg et al. 2010). Ignoring this biological reality can result in overestimation of reversals from uniparental reproduction to outcrossing (Maddison 2006; Goldberg and Igic 2008, 2012; Goldberg et al. 2010). We rudimentarily addressed this issue by preventing this reversal in the Discrete analysis, and our results were qualitatively unchanged. Nonetheless, state-dependent diversification should be incorporated when improved phylogenies and comparative tools become available.

Second, Maddison and FitzJohn (2014) have recently argued that comparative methods for measuring correlations of discrete traits are flawed. When transitions in a trait are rare or concentrated in single lineages, a fundamental assumption of these methods is violated. As a result, coincidence may be mistakenly interpreted as correlation. We find support for many dispersed transitions in both reproductive mode and lifestyle, suggesting that our dataset is relatively robust to Maddison and FitzJohn's (2014) methodological concerns. We cannot, however, reject the possibility that the observed associations are detected for reasons other than correlated evolution.

Third, most nematode species remain undescribed (van Megen et al. 2009; Kiontke et al. 2011). Prior studies of continuous traits suggest that under-sampling itself does not inflate estimates of phylogenetic correlation (Freckleton et al. 2002), but that biased sampling can (Ackerly 2000). Meldal et al. (2007) aimed to sample under-represented taxa for their phylogeny, but the sample of terrestrial taxa likely remains biased: first toward parasites, due to their relevance in public health and agriculture (Meldal et al. 2007; van Megen et al. 2009); second toward uniparental taxa, due to their tractability in the laboratory and overrepresentation in temperate regions where sampling has been concentrated (Bell 1982; Igic and Kohn 2006; van Megen et al. 2009). Oversampling of uniparental and/or parasitic taxa produces an underrepresentation of outcrossing, free-living taxa and thus potentially overestimates the correlation of outcrossing and parasitism. We address this issue in the supplement via simulations to compare true measures of correlation to those obtained from biased subsampling. The simulation results argue that the evolutionary correlations reported here are unlikely to be an artifact of biased sampling of the Nematoda. Biased sampling can weakly inflate correlation estimates under stochastic character mapping but not under the Discrete method. Yet, we find here that several unique tests support significant correlated evolution of outcrossing and parasitism ( $d$ and $m$ in stochastic character mapping; marginal likelihood and model visitation in and model visitation in BayesDiscrete).

The findings we present argue that the Nematoda is one of the most promising phyla in which to investigate the evolutionary and ecological forces underlying the maintenance of outcrossing. Moreover, the diversity of this group allows for an investigation of the mechanisms promoting genetic variation in parasite populations, a subject of the utmost importance (Grant 1994; Castagnone-Sereno 2002; Galvani et al. 2003; De Meeûs et al. 2009; Castagnone-Sereno and Danchin 2014). Until more complete phylogenies become 
available, such investigations should focus upon well-studied subgroups within the Nematoda. The genus of root-knot nematodes Meloidogyne presents an excellent opportunity to examine variation in reproductive mode within an obligately parasitic group (Castagnone-Sereno and Danchin 2014), whereas clades within the suborders Tylenchina and Rhabditina may be valuable for investigating transitions in lifestyle and reproductive mode at a finer scale (De Ley 2006).

\section{Supplementary Material}

Refer to Web version on PubMed Central for supplementary material.

\section{Acknowledgments}

First and foremost, we acknowledge B. Meldal for contributing the sequence and tree data published in Meldal et al. (2007). We thank L. C. Moyle and members of the Moyle lab for access to computing resources. We also thank C. M. Lively, A. L. Dapper, and L. T. Morran for helpful commentary on the manuscript, as well as E. A. Housworth and D. M. Castillo for contributing to the development of this work. We acknowledge many researchers who aided in the assignment of character states to study taxa. These individuals are identified by name in Table S1. We lastly acknowledge the funding sources of Indiana University's research systems: for Big Red II, Lilly Endowment, Inc., the IU Pervasive Technology Institute, and the Indiana METACyt Initiative; for Quarry, NSF grant CNS-0723054. This study was supported by a U.S. National Science Foundation Graduate Research Fellowship and a Common Themes in Reproductive Diversity traineeship (National Institutes of Health funding awarded to Indiana University) to $\mathrm{AKG}$.

\section{LITERATURE CITED}

Plant and Insect Parasitic Nematodes. Nematology Lab at University of Nebraska Lincoln. Available at http://nematode.unl.edu/

Ackerly DD. Taxon sampling, correlated evolution, and independent contrasts. Evolution. 2000; 54:1480-1492. [PubMed: 11108577]

Agrawal AF, Lively CM. Parasites and the evolution of self-fertilization. Evolution. 2001; 55:869-879. [PubMed: 11430647]

Baker HG. Self-compatibility and establishment after 'long-distance' dispersal. Evolution. 1955; 9:347-349.

Barrière A, Félix MA. High local genetic diversity and low outcrossing rate in Caenorhabditis elegans natural populations. Curr. Biol. 2005; 15:1176-1184. [PubMed: 16005289]

Bell, G. The masterpiece of nature: the evolution and genetics of sexuality. Berkeley, CA: University of California Press; 1982.

Billiard S, Lopez-Villavicencio M, Hood ME, Giraud T. Sex, outcrossing and mating types: unsolved questions in fungi and beyond. J. Evol. Biol. 2012; 25:1020-1038. [PubMed: 22515640]

Blaxter M, Ley PDe, Garey J, Liu L, Scheldeman P, Vierstraete A, Vanfleteren J, Mackey L, Dorris M, Frisse L, et al. A molecular evolutionary framework for the phylum Nematoda. Nature. 1998; 392:71-75. [PubMed: 9510248]

Bollback JP. SIMMAP: stochastic character mapping of discrete traits on phylogenies. BMC Bioinformatics. 2006; 7:88-94. [PubMed: 16504105]

Burt A, Bell G. Mammalian chiasma frequencies as a test of two theories of recombination. Nature. 1987; 326:803-805. [PubMed: 3574451]

Carter, K., De Ley, P. Paul's Bizarre Worm Bazaar: a selection of fine worms. 2005. Available at http:// faculty.ucr.edu/ pdeley/bazaar/bazaarhome.htm

Castagnone-Sereno P. Genetic variability of nematodes: a threat to the durability of plant resistance genes? Euphytica. 2002; 124:193-199.

Castagnone-Sereno P. Genetic variability and adaptive evolution in parthenogenetic root-knot nematodes. Heredity. 2006; 96:282-289. [PubMed: 16404412] 
Castagnone-Sereno P, Danchin E. Parasite success without sex - the nematode experience. J. Evol. Biol. 2014; 27:1323-1333. [PubMed: 25105196]

Charlesworth B. The cost of sex in relation to mating system. J. Theor. Biol. 1980; 84:655-671. [PubMed: 7431946]

Criscione CD, Blouin MS. Effective sizes of macroparasite populations: a conceptual model. Trends Parasitol. 2005; 21:212-217. [PubMed: 15837609]

Cutter AD, Wasmuth JD, Washington NL. Patterns of molecular evolution in Caenorhabditis preclude ancient origins of selfing. Genetics. 2008; 178:2093-2104. [PubMed: 18430935]

Dacks J, Roger AJ. The first sexual lineage and the relevance of facultative sex. J. Mol. Evol. 1999; 48:779-783. [PubMed: 10229582]

Darriba D, Taboada G, Doalla R, Posada D. jModelTest 2: more models, new heuristics and parallel computing. Nat. Methods. 2010; 9:772.

De Ley, P. A quick tour of nematode diversity and the backbone of nematode phylogeny. WormBook, ed. The $C$. elegans Research Community, WormBook 1.41.1. 2006. Available at http:// www.wormbook.org/chapters/www_quicktourdiversity/quicktourdiversity.html

De Meeûs, T., Prugnolle, F., Agnew, P. Asexual reproduction in infectious diseases. In: Schön, I.Martens, K., Dijk, P., editors. Lost sex. New York City, NY: Springer; 2009. p. 517-533.

de Vienne DM, Giraud T, Gouyon P-H. Lineage selection and the maintenance of sex. PLoS One. 2013; 8:e66906. [PubMed: 23825582]

Denver DR, Clark KA, Raboin MJ. Reproductive mode evolution in nematodes: insights from molecular phylogenies and recently discovered species. Mol. Phyl. Evol. 2011; 61:584-592.

Dorris M, Ley PDe, Blaxter ML. Molecular analysis of ne-matode diversity and the evolution of parasitism. Parasitol. Today. 1999; 15:188-193. [PubMed: 10322352]

Ferris, H. The nematode-plant expert information system: a virtual encyclopedia on soil and plant nematodes. Davis, CA: University of California; 1999. Available at http://plpnemweb.ucdavis.edu/ nemaplex/index.htm

Fischer O, Schmid-Hempel P. Selection by parasites may increase host recombination frequency. Biol. Lett. 2005; 1:193-195. [PubMed: 17148164]

Fisher, RA. The genetical theory of natural selection. Oxford, U.K: Oxford Univ. Press; 1930.

Fitch, D. Worm Systematic Resource Network. 1998. Available at http://www.nyu.edu/projects/fitch/ WSRN/

Freckleton RP, Harvey PH, Pagel M. Phylogenetic analysis and comparative data: a test and review of evidence. Am. Nat. 2002; 160:712-726. [PubMed: 18707460]

Galvani AP, Coleman RM, Ferguson NM. Antigenic diversity and the selective value of sex in parasites. Ann. Zool. Fenn. 2001; 38:305-314.

Galvani AP, Coleman RM, Ferguson NM. The maintenance of sex in parasites. Proc. R. Soc. B. 2003; 270:19-28.

Gemmil AW, Viney ME, Read AF. Host immune status determines sexuality in a parasitic nematode. Evolution. 1997; 51:393-401.

Goldberg E, Igic B. On phylogenetic tests of irreversible evolution. Evolution. 2008; 62:2727-2741. [PubMed: 18764918]

Goldberg E, Igic B. Tempo and mode in plant breeding system evolution. Evolution. 2012; 66:37013709. [PubMed: 23206129]

Goldberg EE, Kohn JR, Lande R, Robertson KA, Smith SA, Igic B. Species selection maintains selfincompatibility. Science. 2010; 330:493-495. [PubMed: 20966249]

Grant WN. Genetic variation in parasitic nematodes and its implications. Int. J. Parasitol. 1994; 24:821-830. [PubMed: 7982744]

Guindon S, Gascuel O. A simple, fast and accurate method to estimate large phylogenies by maximum-likelihood. Syst. Biol. 2003; 52:696-704. [PubMed: 14530136]

Haldane JBS. Disease and evolution. Ric. Sci. 1949; 19:68-76.

Hamilton WD. Sex versus non-sex versus parasite. Oikos. 1980; 35:282-290.

Hamilton WD, Axelrod R, Tanese R. Sexual reproduction as an adaptation to resist parasites (a review). Proc. Natl. Acad. Sci. USA. 1990; 87:3566-3573. [PubMed: 2185476] 
Hartfield M, Otto SP, Keightley PD. The role of advantageous mutations in enhancing the evolution of a recombination modifier. Genetics. 2010; 184:1153-1164. [PubMed: 20139345]

Hartfield M, Otto SP, Keightley PD. The maintenance of obligate sex in finite, structured populations subject to recurrent beneficial and deleterious mutations. Evolution. 2012; 66:3658-3669. [PubMed: 23206126]

Hedrick PW. Evolutionary genetics of the major histocompatibility complex. Am. Nat. 1994; 143:945964.

Howard RS, Lively CM. Parasitism, mutation accumulation and the maintenance of sex. Nature. 1994; 367:554-557. [PubMed: 8107824]

Howard RS, Lively CM. The ratchet and the Red Queen: the maintenance of sex in parasites. J. Evol. Biol. 2002; 15:648-656.

Huelsenbeck JP, Ronquist F. MrBayes: Bayesian inference of phylogeny. Bioinformatics. 2001; 17:754-755. [PubMed: 11524383]

Huelsenbeck JP, Nielsen R, Bollback JP. Stochastic mapping of morphological characters. Syst. Biol. 2003; 52:131-158. [PubMed: 12746144]

Hurst LD, Peck JR. Recent advances in understanding of the evolution and maintenance of sex. Trends Ecol. Evol. 1996; 11:46-52. [PubMed: 21237760]

Igic B, Kohn JR. The distribution of plant mating systems: study bias against obligately outcrossing species. Evolution. 2006; 60:1098-1103. [PubMed: 16817548]

Igic B, Bohs L, Kohn JR. Ancient polymorphism reveals unidirectional breeding system shifts. Proc. Natl. Acad. Sci. USA. 2006; 103:1359-1363. [PubMed: 16428289]

Jaenike J. An hypothesis to account for the maintenance of sex within populations. Evol. Theor. 1978; 3:191-194.

Kass RE, Raftery AE. Bayes factors. J. Am. Stat. Assoc. 1995; 90:773-795.

Keightley PD, Otto SP. Interference among deleterious mutations favours sex and recombination in finite populations. Nature. 2006; 443:89-92. [PubMed: 16957730]

King KC, Jokela J, Lively CM. Trematode parasites infect or die in snail hosts. Biol. Lett. 2011; 7:265-268. [PubMed: 20961880]

Kiontke, K., Fitch, DHA. The phylogenetic relationships of Caenorhabditis and other rhabditids. WormBook, ed. The $C$. elegans Research Community. 2005. Available at http:// www.wormbook.org/chapters/www_phylogrhabditids/phylorhab.html

Kiontke K, Gavin NP, Raynes Y, Roehrig C, Piano F, Fitch DH. Caenorhabditis phylogeny predicts convergence of hermaphroditism and extensive intron loss. Proc. Natl. Acad. Sci. USA. 2004; 101:9003-9008. [PubMed: 15184656]

Kiontke K, Felix MA, Ailion M, Rockman M, Braendle C, Peni-gault J-B, Fitch D. A phylogeny and molecular barcodes for Caenorhabditis, with numerous new species from rotting fruits. BMC Evol. Biol. 2011; 11:339-356. [PubMed: 22103856]

Lajeunesse MJ, Forbes MR. Host range and local parasite adaptation. Proc. R. Soc. B. 2002; 269:703710.

Lively CM. Evidence from a New Zealand snail for the maintenance of sex by parasitism. Nature. 1987; 328:519-521.

Lively CM, Lloyd DG. The cost of biparental sex under individual selection. Am. Nat. 1990; 135:489500.

Lively CM, Morran LT. The ecology of sexual reproduction. J. Evol. Biol. 2014; 27:1292-1303. [PubMed: 24617324]

Lynch M, Bürger R, Butcher D, Gabriel W. The mutational meltdown in asexual populations. Heredity. 1993; 84:339-344.

Lythgoe KA. The coevolution of parasites with host-acquired immunity and the evolution of sex. Evolution. 2000; 54:1142-1156. [PubMed: 11005284]

Maddison WP. Confounding asymmetries in evolutionary diversification and character change. Evolution. 2006; 60:1743-1746. [PubMed: 17017073]

Maddison, W., Maddison, D. Mesquite: a modular system for evolutionary analysis.. Version 2.75. 2011. Available at http://mesquiteproject.org 
Maddison WP, FitzJohn RG. The unsolved challenge to phylogenetic correlation tests for categorical characters. Syst. Biol. 2014; 64:127-136. [PubMed: 25209222]

Maupas E. Modes et formes de reproduction des nematodes. Arch. Zool. Exp. Gen. 1900; 3:463-624.

Maynard Smith, J. The evolution of sex. Cambridge, U.K: Cambridge Univ. Press; 1978.

Meldal B, Debenham N, Ley PDe, Ley IDe, Vanfleteren J, Vierstraete A, Bert W, Borgonie G, Moens T, Tyler P, et al. An improved molecular phylogeny of the Nematoda with special emphasis on marine taxa. Mol. Phyl. Evol. 2007; 42:622-636.

Muller HJ. Some genetic aspects of sex. Am. Nat. 1932; 66:118-138.

Muller HJ. The relation of recombination to mutational advance. Mutat. Res. 1964; 1:2-9.

Nadler SA. Microevolution and the genetic structure of parasite populations. J. Parasitol. 1995; 81:395-403. [PubMed: 7776124]

Newton M, Raftery AE. Approximate Bayesian inference with the weighted likelihood bootstrap. J. R. Stat. Soc. B Stat. Methodol. 1994; 56:3-48.

Nielsen R. Mapping mutations on phylogenies. Syst. Biol. 2002; 51:729-739. [PubMed: 12396587]

Nunney L. The maintenance of sex by group selection. Evolution. 1989; 43:245-257.

Obbard DJ, Jiggins FM, Halligan DL, Little TJ. Natural selection drivese extremely rapid evolution in antiviral RNAi genes. Curr. Biol. 2006; 16:580-585. [PubMed: 16546082]

Otto SP. The evolutionary enigma of sex. Am. Nat. 2009; 174:S1-S14. [PubMed: 19441962]

Otto SP, Barton NH. Selection for recombination in small populations. Evolution. 2001; 55:19211931. [PubMed: 11761054]

Pagel M. Detecting correlated evolution on phylogenies: a general method for the comparative analysis of discrete characters. Proc. R. Soc. B. 1994; 255:37-45.

Pagel M, Meade A. Bayesian analysis of correlated evolution of discrete characters by reversible-jump Markov chain Monte Carlo. Am. Nat. 2006; 167:808-825. [PubMed: 16685633]

Pannell JR. The evolution and maintenance of androdioecy. Ann. Rev. Ecol. Syst. 2002; 33:397-425.

Paterson S, Vogwill T, Buckling A, Benmayor R, Spiers AJ, Thomson NR, Quail M, Smith F, Walker D, Libberton B. Antagonistic coevolution accelerates molecular evolution. Nature. 2010; 464:275278. [PubMed: 20182425]

R Core Team. R: a language and environment for statistical computing. Vienna, Austria: R Foundation for Statistical Computing; 2013. Available at http://www.R-project.org

Raftery, AE., Newton, MA., Satagopan, JM., Krivitsky, PN. Working Paper no. 60R, Center for Statistics and Social Sciences. Seattle, WA: University of Washington; 2006. Estimating the integrated likelihood via posterior simulation using the harmonic mean identity.

Ross L, Hardy NB, Okusu A, Normark BB. Large population size predicts the distribution of asexuality in scale insects. Evolution. 2013; 67:196-206. [PubMed: 23289572]

Salathé M, Kouyos RD, Regoes RR, Bonhoeffer S. Rapid parasite adaptation drives selection for high recombination rates. Evolution. 2008; 62:295-300. [PubMed: 18039325]

Schliep K. phangorn: phylogenetic analysis in R. Bioinformatics. 2011; 27:592-593. [PubMed: 21169378]

Schulte RD, Makus C, Hasert B, Michiels NK, Schulenburg H. Multiple reciprocal adaptations and rapid genetic change upon experimental coevolution of an animal host and its microbial parasite. Proc. Natl. Acad. Sci. USA. 2010; 107:7359-7364. [PubMed: 20368449]

Schulte RD, Makus C, Schulenburg H. Host-parasite coevolution favours parasite genetic diversity and horizontal gene transfer. J. Evol. Biol. 2013; 26:1836-1840. [PubMed: 23865952]

Schultz TR, Churchill GA. The role of subjectivity in reconstructing ancestral character states: a Bayesian approach to unknown rates, states, and transformation asymmetries. Syst. Biol. 1999; 48:651-664.

Suomalainen, E., Saura, A., Lokki, J. Cytology and evolution in parthenogenesis. Boca Raton, FL: CRC Press, Inc; 1987.

Tamura K, Peterson D, Peterson N, Stecher G, Nei M, Kumar S. MEGA5: molecular evolution genetics analysis using maximum likelihood, evolutionary distance, and maximum parsimony methods. Mol. Biol. Evol. 2011; 28:2731-2739. [PubMed: 21546353] 
Thompson JN. Specific hypotheses on the geographic mosaic of coevolution. Am. Nat. 1999; 153:S1S14.

Triantaphyllou A, Hirschmann H. Reproduction in plant and soil nematodes. Ann. Rev. Phytopathol. 1964; 2:57-80.

van Megen H, van den Elsen S, Holterman M, Karssen G, Mooyman P, Bongers T, Holovachov O, Bakker J, Helder J. A phylogenetic tree of nematodes based on about 1200 full-length small subunit ribosomal DNA sequences. Nematology. 2009; 11:927-950.

Van Valen L. Molecular evolution as predicted by natural selection. J. Mol. Evol. 1974; 3:89-101. [PubMed: 4407466]

Verhoeven KJF, Biere A. Geographic parthenogenesis and plant-enemy interactions in the common dandelion. BMC Evol. Biol. 2013; 13:23-30. [PubMed: 23356700]

Weeks SC, Benvenuto C, Reed SK. When males and hermaphrodites coexist: a review of androdioecy in animals. Integr. Comp. Biol. 2006; 46:449-464. [PubMed: 21672757]

West SA, Gemmil AW, Graham AL, Viney ME, Read AF. Immune stress and facultative sex in a parasitic nematode. J. Evol. Biol. 2001; 14:333-337.

Williams, GC. Sex and evolution. Princeton, NJ: Princeton Univ. Press; 1975.

Wilson CG, Sherman PW. Spatial and temporal escape from fungal parasitism in natural communities of anciently asexual bdelloid rotifers. Proc. R. Soc. B. 2013; 280:1255-1262.

Zhan J, Mundt CC, McDonald BA. Sexual reproduction facilitates the adaptation of parasites to antagonistic host environments: evidence from empirical studyinthe wheat-Mycosphaerella graminicola system. Int. J. Parasitol. 2007; 37:861-870. [PubMed: 17451717] 


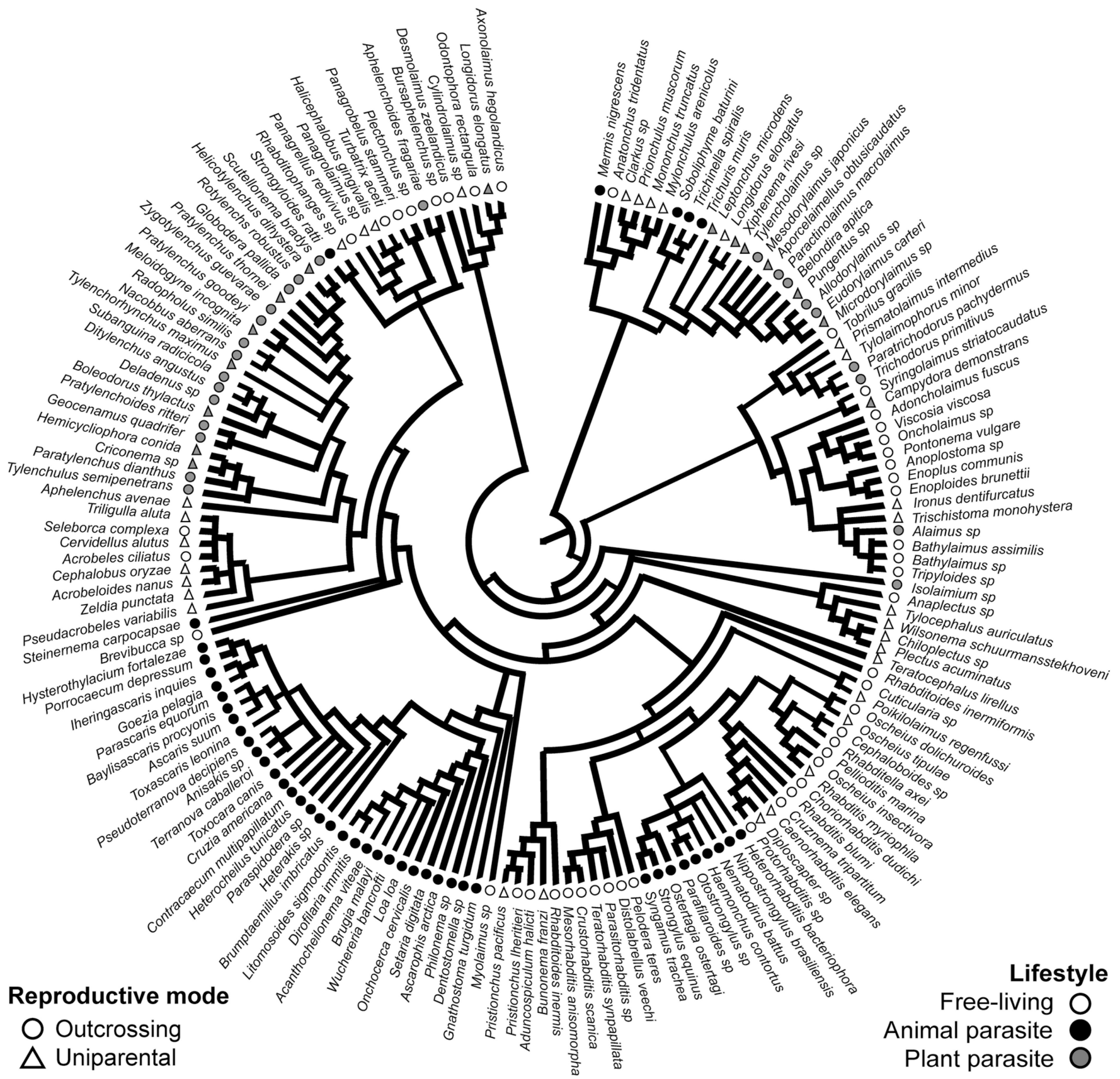

Figure 1.

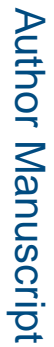

Majority-rule consensus tree and character distribution of 162 species in the Nematoda. This reconstruction represents the consensus of 2700 Bayesian-inferred trees (modified from Meldal et al. 2007). Pruning of the original tree is described in the Methods section. Further modifications were performed in MEGA 5.1 (Tamura et al. 2011). Symbol fill indicates lifestyle: free living (open), animal parasite (black), and plant parasite (gray). Symbol shape indicates reproductive mode: outcrossing (circle) and uniparental (triangle). 

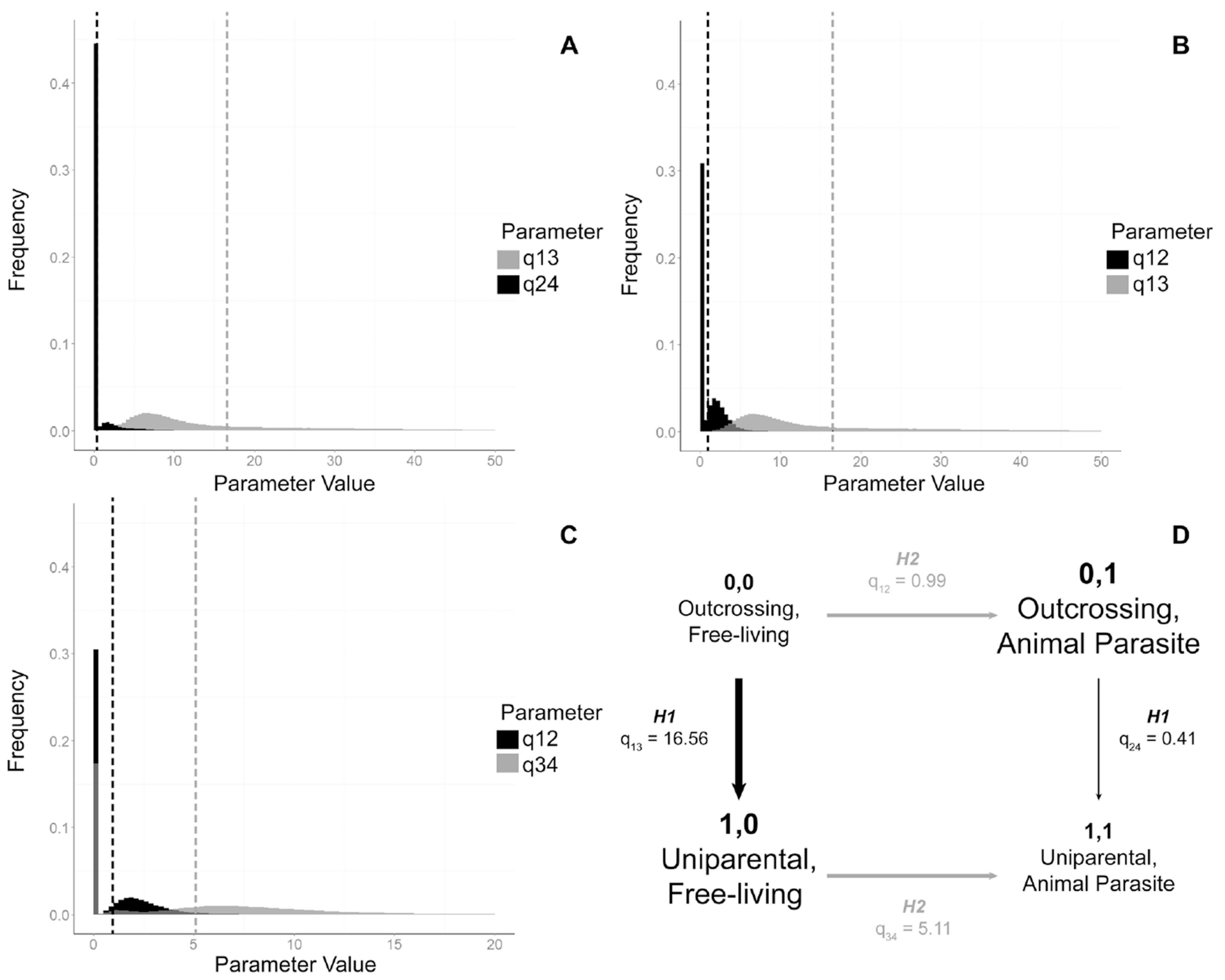

C
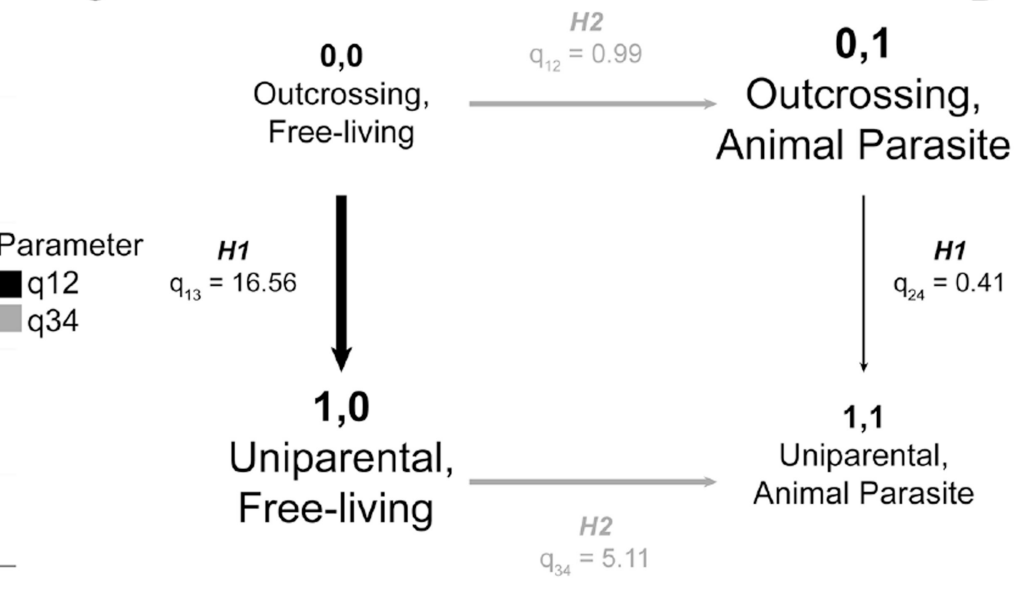

Figure 2.

Estimated evolutionary transition rates in reproductive mode and lifestyle. (A-C) Posterior probability distribution of the values of transition rate parameters as estimated in one representative run of five dependent analyses in BayesDiscrete. Comparisons between two different transition rate distributions are displayed to test specific hypotheses. (A) $\mathrm{H} 1$ : the transition rate from outcrossing to uniparental reproduction is greater on a free living $\left(\mathrm{q}_{13}\right)$ than on an animal parasitic background $\left(\mathrm{q}_{24}\right)$. (B) $\mathrm{H} 1$ : transition rates in reproductive mode ( $\mathrm{q}_{13}$ ) exceed those in lifestyle $\left(\mathrm{q}_{12}\right)$. (C) $\mathrm{H} 2$ : the transition rate from free living to animal parasitism is identical on outcrossing $\left(\mathrm{q}_{12}\right)$ and uniparental backgrounds $\left(\mathrm{q}_{34}\right)$. Dotted lines indicate the mean estimated transition rate for the corresponding parameter. (D) A diagram of investigated evolutionary transitions between the four different character states for reproductive mode and lifestyle. Larger type indicates character states for which a statistical excess of taxa is found. Line weight corresponds to the magnitude of the transition rate, estimated as the average value across five runs of the dependent analysis in BayesDiscrete. Black indicates transitions compared to test Hypothesis 1 and gray indicates Hypothesis 2. 

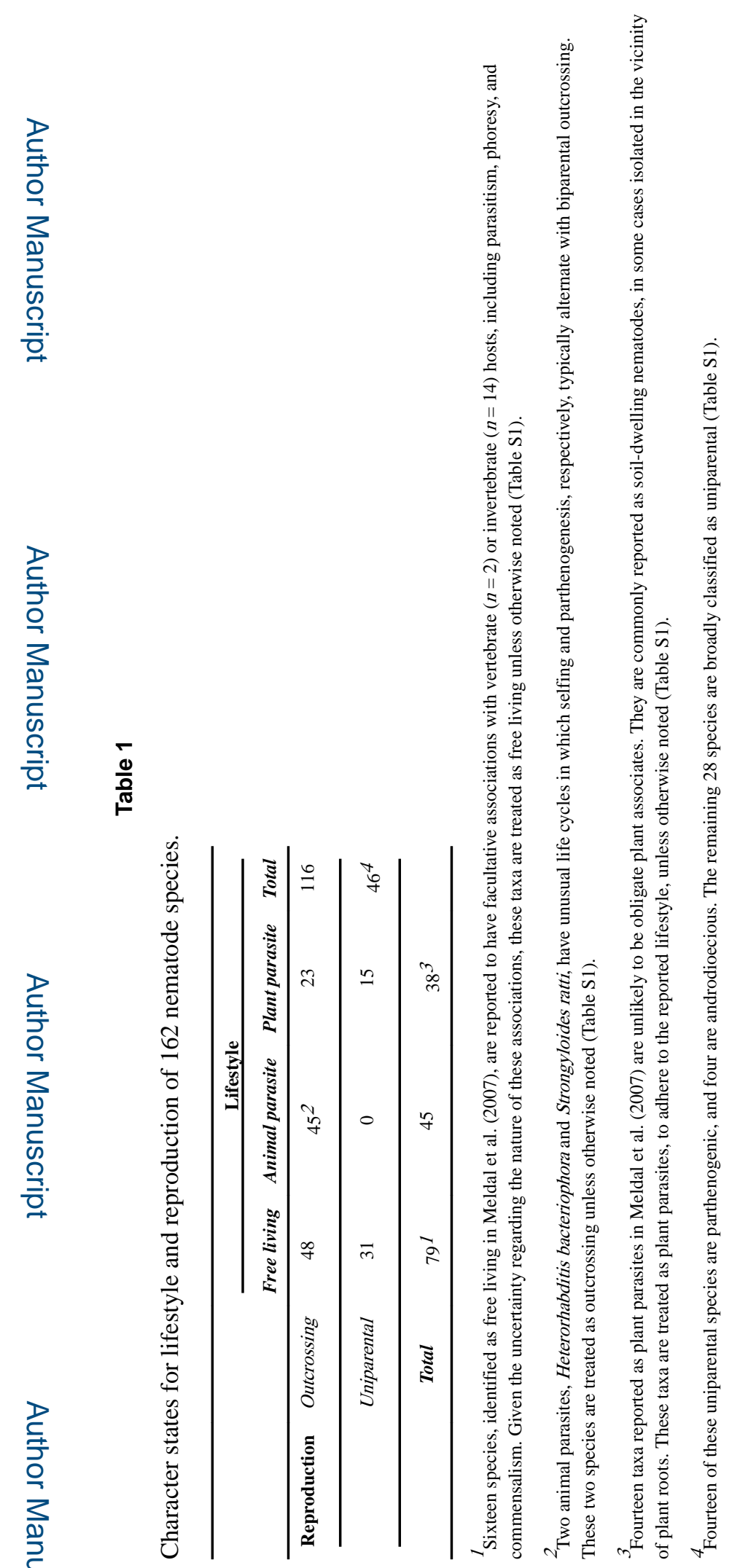

Evolution. Author manuscript; available in PMC 2017 February 23. 